\title{
Tetrazolium-based cytotoxicity assay to determine anti-protozoal activity against the scuticociliate Anophryoides haemophila
}

\author{
Alastair E. Cribb ${ }^{1}$, Beatrice Despres ${ }^{2}$, Richard J. Cawthorn ${ }^{2, *}$ \\ Lobster Health Research Centre, ${ }^{1}$ Departments of Anatomy and Physiology, and \\ ${ }^{2}$ Microbiology and Pathology, Atlantic Veterinary College, University of Prince Edward Island, Charlottetown, \\ Prince Edward Island, C1A 4P3, Canada
}

\begin{abstract}
Anophryoides haemophila is a ciliated protozoan that causes 'bumper car' disease in American lobster Homarus americanus. Currently, there are no effective pharmaceuticals approved for use to treat the disease, which can be a significant source of mortality in lobster holding facilities. We have modified a tetrazolium-based cytotoxicity assay to determine the anti-protozoal activity of compounds towards $A$. haemophila. To obtain reproducible results, A. haemophila maintained in continuous culture were preferable to freshly isolated A. haemophila. It was also found that the ciliates must be replicating to produce reproducible results. Maximal anti-protozoal activity was evident after a $24 \mathrm{~h}$ incubation period. Oxytetracycline was found to be relatively inactive, while high concentrations ( $1 \mathrm{mM}$ ) of formaldehyde and sulphaquinoxaline were required to produce $50 \%$ cytotoxicity. In contrast, $100 \mu \mathrm{M}$ lasalocid produced $92 \pm 4 \%$ and $100 \mu \mathrm{M}$ pyrimethamine produced $84 \pm 6.5 \%$ cytotoxicity after $24 \mathrm{~h}$. This objective assay provides a rapid means of screening compounds to identify those with potential in vivo activity against Anophryoides haemophila.
\end{abstract}

KEY WORDS: Drug - Anophryoids haemophila . Protozoa - Lobster ' In vitro - Tetrazolium - Antiprotozoal

\section{INTRODUCTION}

Anophryoides haemophila sp. $\mathrm{n}$. is a ciliated protozoan that causes 'bumper car' disease in American lobster Homarus americanus (Cawthorn et al. 1996). Bumper car disease can be a source of significant mortality in lobsters maintained in holding facilities at cold temperatures (Cawthorn 1997). No effective, approved chemotherapeutants exist for the control or treatment of bumper car disease. A rapid, effective screening assay that could be employed to quickly identify compounds with activity against $A$. haemophila would facilitate identification of potential candidate compounds.

We had previously developed a simple in vitro system based on light microscopic observation of ciliate motility and morphology to assess the viability of the

\footnotetext{
-Addressee for correspondence. E-mail: cawthorn@upei.ca
}

parasite when exposed to various compounds (Novotny et al. 1996). Using this system, we observed that monensin, formaldehyde, and pyrimethamine+sulphaquinoxaline caused dose-dependent reduction in ciliate motility and morphological changes. Amprolium, a coccidiostat, was ineffective, while oxytetracycline, an antibiotic approved for the treatment and prevention of gaffkemia in lobsters, produced variable results. This earlier method, while useful for a preliminary screen, has several disadvantages. It is laborious and assessment of effects on the ciliate is somewhat subjective. Most importantly, we have subsequently observed that loss of motility of the ciliate is not necessarily an indication of death (Despres, Cribb \& Cawthorn unpubl. obs.).

Tetrazolium-based assays are commonly used to assess cytotoxicity in mammalian cell systems (Marshall et al. 1995) and to measure viability in aquatic protozoal parasites (Dungan \& Hamilton 1995). The 
exact metabolic pathway responsible for the conversion of the tetrazolium compounds to coloured formazan products that can be measured spectrophotometrically varies with the specific compound and to some extent the cell type (Vistica et al. 1991, Marshall et al. 1995). We selected a commercially available assay based on the formation of soluble formazan products from the tetrazolium compound 3-(4,5-dimethylthiazol-2-yl)-5-(3-carboxymethoxyphenyl)-2-(4sulfophenyl)-2H-tetrazolium (MTS, Owen's reagent) in the presence of an electron coupling reagent, PMS (phenazine methosulfate). Because the resulting formazan product is soluble, its density can be measured directly on the spectrophotometer without intervening centrifugation or solubilization steps

We used a panel of potential anti-protozoal compounds to develop this assay for use with Anophryoides hacmophila. The compounds were selected for their different mechanisms of action and previously demonstrated ability (or inability) to inhibit growth of A. haemophila or other protozoal parasites. They were used primarily to explore assay parameters and not necessarily as desirable therapeutic compounds in vivo.

\section{MATERIALS AND METHODS}

Source of protozoa. In experiments involving freshly isolated Anophryoides haemophila, protozoa were obtained from previously infected lobsters maintained at $2^{\circ} \mathrm{C}$ in the Aquatic Animal Facility at the Atlantic Veterinary College (AVC Animal Care Committee) and isolated as previously described (Novotny et al. 1996). The protozoa were used immediately.

Anophryoides haemophila were also maintained in continuous culture in a cell-free, defined medium at $4^{\circ} \mathrm{C}$ (Lee \& Soldo 1992, Messick \& Small 1996). This artificial seawater-based medium was supplemented with vitamins (RPMI 1640 vitamins solution, Sigma, Oakville, Ontario), 10\% fetal bovine serum, and penicillin/streptomycin $\left(100 \mathrm{U} \mathrm{ml}^{-1} ; 100 \mathrm{\mu g} \mathrm{ml}^{-1}\right)$. All reagents used in the culture medium were obtained from commercial sources.

Prior to use, Anophryoides haemophila obtained by either method were collected by centrifugation at approximately $400 \times g$ for $5 \mathrm{~min}$ at $4^{\circ} \mathrm{C}$ and re-suspended at appropriate concentrations in the culture medium immediately prior to use.

Cytotoxicity assay. The cytotoxicity assay was performed with reagents obtained from Promega (Cell Titer $96 \mathrm{AQ}_{\text {ueous }}$ Non-radioactive Cell Proliferation Assay, Promega, Madison, WI). This assay depends on the formation of soluble formazan products from the tetrazolium compound 3-(4,5-dimethylthiazol-2-yl)-5(3-carboxymethoxyphenyl)-2-(4-sulfophenyl)-2H-tetra- zolium (MTS) in the presence of an electron coupling reagent, PMS (phenazine methosulfate). The reagents were diluted as recommended by the manufacturer to give a final concentration of $2 \mathrm{mg} \mathrm{ml}^{-1}$ MTS and $0.045 \mathrm{mg} \mathrm{ml}^{-1}$ PMS

After isolation and counting, the protozoa were aliquoted at the optimum plating density as determined in the experiments described below in a volume of $75 \mu$. These experiments determined the ciliate concentration and incubation times that resulted in continual growth of the ciliates and a linear relationship between protozoal number and optical density (OD).

After a stabilization period of $1 \mathrm{~h}$ at $4^{\circ} \mathrm{C}$, test compounds were added in $25 \mu \mathrm{l}$ of medium. All test compounds were obtained from Sigma Chemical Co., Oakville, Ontario. Drugs were prepared by dissolving directly in the medium (formaldehyde, sulphaquinoxaline, oxytetracyclinej or in dimethyl sulfoxide (pyrimethamine, lasalocid) prior to dissolving in medium. The final concentration of dimethyl sulfoxide never exceeded $1 \%$ in the wells. This concentration did not have any effect on viability of the ciliates.

After an appropriate incubation period ( 0 to $45 \mathrm{~h}$, depending on the particular experimental conditions), $20 \mu \mathrm{l}$ of the PMS/MTS mixture were added to each well. The plates were incubated for an additional $3 \mathrm{~h}$ at $4^{\circ} \mathrm{C}$ and then the $O D$ was measured at $490 \mathrm{~nm}$ with a microplate reader. In general, all conditions were performed with 6 replicates per plate, and the mean from each set of replicates was used in all subsequent calculations. Results are presented, unless specified otherwise, as the mean \pm standard error of 3 replicate experiments

The background OD was determined by a combination of medium alone, medium plus ciliates, and medium plus MTS. All plates contained appropriate test compound controls to ensure there were no direct interactions between the drugs and MTS. No test compounds influenced the background OD reading or had any direct effect on the MTS conversion. The background $O D$ was subtracted from all values obtained prior to any calculations of cytotoxicity.

Cytotoxicity was determined according to the following formula:

$$
\% \text { cytotoxicity }=\frac{\text { control OD }- \text { treatment } O D}{\text { control OD }} \times 100 \%
$$

The control OD is determined in the wells that contain no added drug, at the time the experiment is terminated. Thus, a lower OD reading in the treated wells reflects a decreased number of viable ciliates and hence an increased cytotoxicity. Because the ciliates may be replicating during the time of the experiment, this measure of cytotoxicity encompasses both inhibition of replication (cytostatic effects) and actual death of protozoa (cytocidal effects). 
Determination of replication during the experimental period is based on comparison of the baseline $O D$ in a set of control wells determined at the time when drugs were added and the OD in the control (no treatment) wells at the end of the experimental period. An increase in $O D$ in the control wells over the course of the experimental period indicates that the ciliates were replicating (that is, an increase in number of ciliates per well).

The time of the reported data point always reflects the time at which OD was measured, although the addition of the tetrazolium reagent occurred $3 \mathrm{~h}$ previously. Thus, the first time point that could be measured is $3 \mathrm{~h}$ postaddition of the drug and tetrazolium reagent.

\section{RESULTS}

\section{Determination of assay conditions}

The MTS/PMS assay has previously been employed in cells and organisms held at ambient temperatures and above (Dungan \& Hamilton 1995, Marshall et al. 1995). The growth characteristics of Anophryoides haemophila required that the assay be conducted at $4^{\circ} \mathrm{C}$. Therefore, we initially had to determine that the metabolic pathways responsible for formation of the formazan product existed in A. haemophila and were active at this temperature. As shown in Fig. 1, ciliates maintained in culture, and those obtained directly from infected lobsters, had the ability to produce the

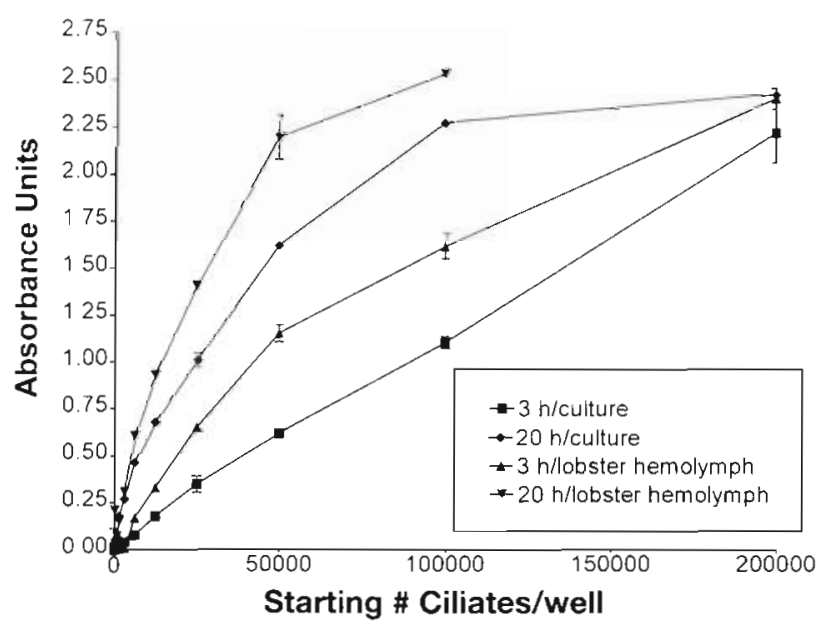

Fig. 1. Anophyroides haemophila. Relationship between number of ciliates per well and optical density of the formazan product. Ciliates isolated from lobster hemolymph or maintained in culture were aliquoted at varying concentrations into wells of a 96-well plate. MTS/PMS (see 'Materials and methods: cytotoxicity assay') was added immediately or after $17 \mathrm{~h}$ of incubation, and the optical density was read $3 \mathrm{~h}$ later ( 3 and $20 \mathrm{~h}$ time points, respectively). Results are presented as mean $\pm \mathrm{SD}$

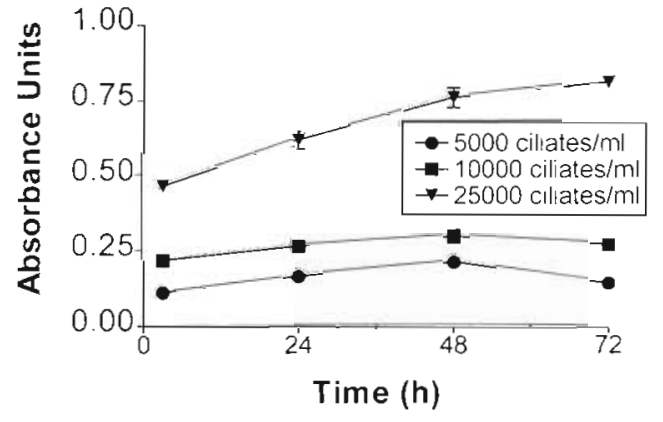

Fig. 2. Anophyroides haemophila. Growth curve of ciliates in culture. Ciliates were aliquotted at 5, 10, and 25 thousand ciliates per well. After a $3 \mathrm{~h}$ incubation with MTS/PMS, formation of formazan was determined at 24,48 , and $72 \mathrm{~h}$ after initial plating. Results are presented as mean $\pm \mathrm{SD}$

soluble formazan product. The relationship between ciliate number and OD was linear up to 200000 ciliates per well if assayed immediately. However, with time in culture, the ciliates multiplied and linearity was lost significantly when starting ciliate numbers exceeded 50000 per well prior to the $20 \mathrm{~h}$ incubation. When ciliates were maintained for up to $72 \mathrm{~h}$ at a concentration of 25000 ciliates per well, linearity was maintained throughout the experimental period (Fig. 2). Linearity of growth was lost after $48 \mathrm{~h}$ at lower ciliate concentrations and, further, growth was superior at a starting concentration of 25000 ciliates per well. Therefore, a concentration of 25000 ciliates per well and an incubation period not exceeding $48 \mathrm{~h}$ in the 96 -well plates was selected for the following experiments.

To determine the optimum exposure periods for assessing the effects of compounds, we determined viability with MTS/PMS at various times after exposure. Formaldehyde was selected as an initial model compound because it was reported to be cytotoxic towards Anophryoides haemophila (Novotny et al. 1996). Ciliates harvested directly from lobsters were employed in the first series of experiments, but there were marked differences in the apparent efficacy of formaldehyde depending on whether the ciliates were multiplying (as evidenced by an increase in the OD in the control wells at $24 \mathrm{~h}$ compared to the OD at $3 \mathrm{~h}$ ). As shown in Fig. 3, when the protozoa were not growing, cytotoxicity was markedly decreased at $24 \mathrm{~h}$. Note that the cytotoxicity at $3 \mathrm{~h}$ (i.e. simultaneous addition of formaldehyde and the tetrazolium reagents) was much less than at $24 \mathrm{~h}$. The delayed onset of toxicity and the effect of growth of the ciliate on apparent toxicity were observed with several compounds (data not shown). Variability in growth was particularly apparent when A. haemophila were harvested directly from lobsters and may have been related to the stage of infection and overall health of the lobster. Because of this variable growth rate which affected the apparent efficacy 

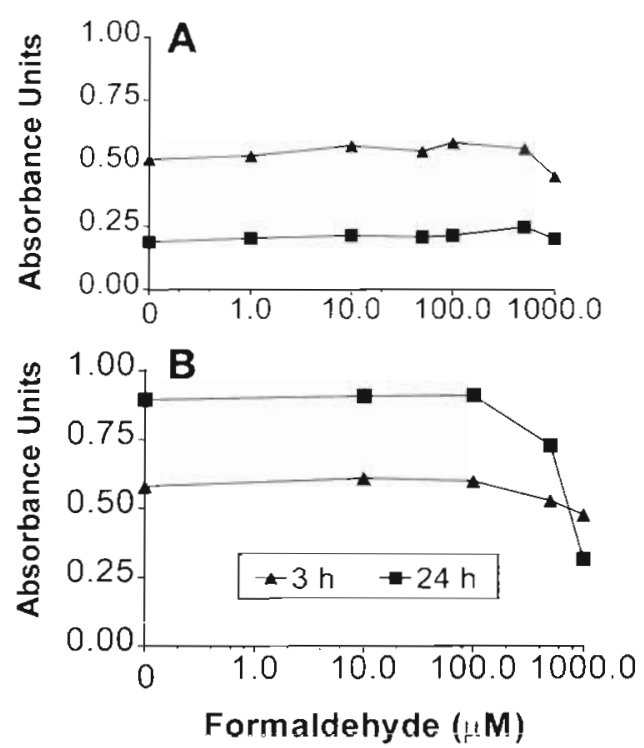

Fig. 3. Effect of formaldehyde on viability of growing and non-growing ciliates. Ciliates were harvested directly from infected lobster hemolymph on 2 separate days and aliquotted at 25000 ciliates per well. Formaldehyde at various concentrations was added. Viability was determined at $3 \mathrm{~h}$ and $24 \mathrm{~h}$ based on MTS conversion. (A) In a first experiment, ciliates did not replicate during the $24 \mathrm{~h}$ incubation period (note that the $\mathrm{OD}$ at $3 \mathrm{~h}$ in the no-formaldehyde $[0 \mu \mathrm{M}]$ wells is higher than the OD at $24 \mathrm{~h}$ in the comparable wells) and no significant toxicity of formaldehyde was observed. (B) In a second experiment, the ciliates were replicating over the $24 \mathrm{~h}$ incubation period (compare the OD at $3 \mathrm{~h}$ and $24 \mathrm{~h}$ in the noformaldehyde $[0 \mu \mathrm{M}]$ wells) and significant anti-protozoal effects were observed at $24 \mathrm{~h}$, but not at $3 \mathrm{~h}$

of the compounds in vitro, all subsequent studies were performed with A. haemophila maintained in culture. The results obtained with cultured ciliates were similar to, but more consistently reproducible than those obtained using ciliates obtained directly from lobsters.

The following conditions were used in subsequent experiments with compounds having potential efficacy against Anophryoides haemophila: (1) A. haemophila was incubated in $100 \mu \mathrm{l}$ of medium at 25000 ciliates per well. (2) Baseline formazan formation at $3 \mathrm{~h}$ was measured to verify continued replication during the experimental period. (3) Viability was determined by formazan formation after 24 and 48 h of exposure. (4) All experiments were repeated 3 times; results are presented as mean $\pm \mathrm{SE}$.

The effects of formaldehyde on Anophryoides haemophila under these conditions are shown in Fig. 4. After an incubation period of $24 \mathrm{~h}$, concentrations of formaldehyde greater than $500 \mu \mathrm{M}$ showed marked cytotoxicity, with concentrations of $5 \mathrm{mM}$ and above killing $90 \pm 5 \%$ of the ciliates at $24 \mathrm{~h}$. An apparently aberrant increase in viability with $500 \mu \mathrm{M}$ formaldehyde was observed at $48 \mathrm{~h}$. Formaldehyde itself had no direct

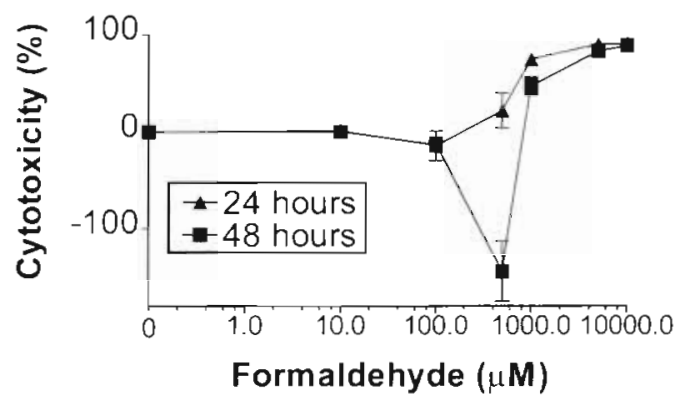

Fig. 4. Anophyroides haemophila. Anti-protozoal effects of formaldehyde on ciliates as assessed by formazan formation under standardized conditions (see 'Results: determination of assay conditions')

effect on the conversion of MTS to formazan at any concentration (data not shown).

Oxytetracycline up to concentrations of $1 \mathrm{mM}$ had only limited effects on Anophryoides haemophila, reaching a maximum cytotoxicity of only $35 \%$ at $48 \mathrm{~h}$ (Fig. 5). There was very little difference between 24 and $48 \mathrm{~h}$ drug exposure periods.

Sulphaquinoxaline was selected as a representative of the sulfonamide anti-protozoal group of compounds. Usually this drug is used in combination with pyrimethamine, a dihydropyrimidine compound with activity against coccidia. These compounds were initially tested singly and then in combination. Sulphaquinoxaline had no immediate effect on the ciliates ( $3 \mathrm{~h}$ incubation; data not shown), but displayed modest antiprotozoal effects at 24 and $48 \mathrm{~h}$ (Fig. 6). Pyrimethamine had no immediate effects (data not shown), but displayed marked anti-protozoal effects at concentrations of 50 and $100 \mu \mathrm{M}$ at 24 and $48 \mathrm{~h}$ (Fig. 6). As shown in Fig. 7 , there were no apparent synergistic effects between pyrimethamine and sulfaquinoxaline.

Lasalocid is a divalent polyether ionophore with activity against coccidia. It also showed significant anti-protozoal activity at concentrations of 50 and $100 \mu \mathrm{M}$ (Fig. 8).

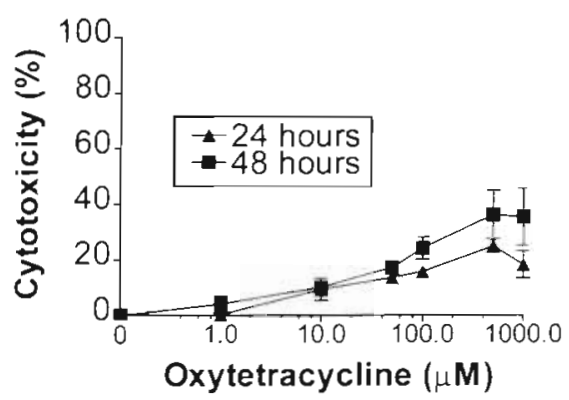

Fig. 5. Anophyroides haemophila. Anti-protozoal effects of oxytetracyline on ciliates as assessed by formazan formation under standardized conditions (see 'Results: determination of assay conditions') 

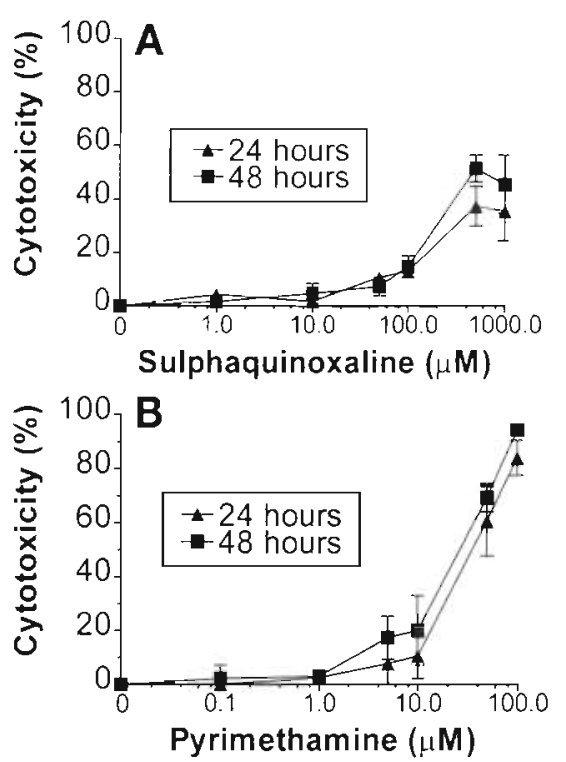

Fig. 6. Anophyroides haemophila. Anti-protozoal effects of sulphaquinoxaline (A) and pyrimethamine (B) on ciliates as assessed by formazan formation under standardized conditions (see 'Results: determination of assay conditions')
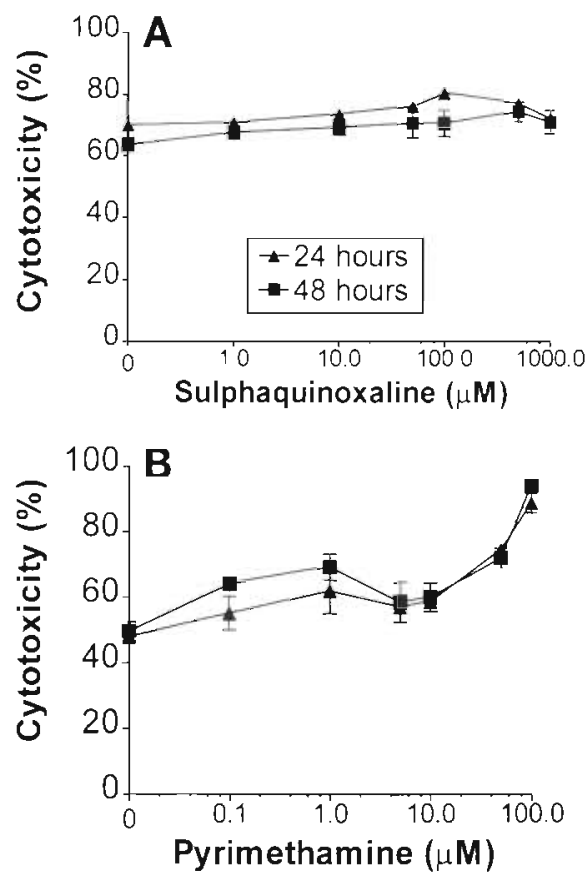

Fig. 7. Lack of synergistic effects between pyrimethamine and sulphaquinoxaline. (A) Pyrimethamine $(50 \mu \mathrm{M})$ incubated with increasing concentrations of sulphaquinoxaline. When pyrimethamine was added in increasing concentrations to sulphaquinoxaline $(500 \mu \mathrm{M})$, toxicity increased but not above that observed with pyrimethamine alone (compare with Fig. 6). (B) Sulphaquinoxaline did not increase the toxicity observed with pyrimethamine alone

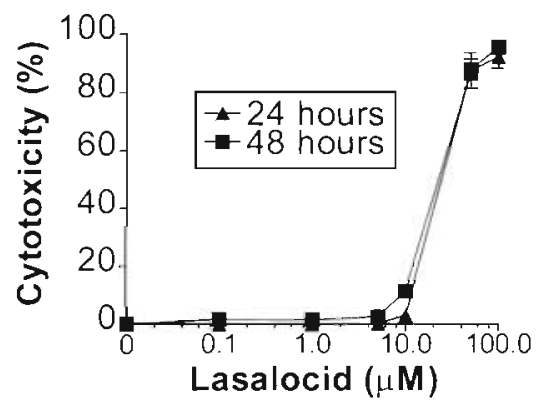

Fig. 8. Anophyroides haemophila. Anti-protozoal effects of lasalocid on ciliates as assessed by formazan formation under standardized conditions (see 'Results: determination of assay conditions')

\section{DISCUSSION}

These results demonstrated the utility of an in vitro tetrazolium-based assay in assessing activity of compounds against the lobster parasite Anophyroides haemophila. We previously identified $A$. haemophila as the cause of bumper car disease in lobsters (Cawthorn et al. 1996). The ability to maintain the isolated parasite in a cell-free defined medium based on artificial seawater ensures a readily available, consistent source of $A$, haemophila for use in in vitro assays. Additionally, we are able to infect lobsters with the ciliate and obtain large numbers of the parasite from the host hemolymph.

Several interesting technical points relating to the use of this protozoa in an in vitro toxicity assay were discovered in these experiments. First, it is necessary to ensure that the Anophryoides haemophila are viable and replicating when the assay is performed, or the cytotoxic potential of a compound is markedly underestimated. This requirement essentially precluded the use of freshly isolated protozoa in the experimental protocol. Secondly, a time period of approximately $24 \mathrm{~h}$ was required to observe the maximal effect of compounds on the ciliate, providing a useful time period for screening experiments.

We were able to hold the parasite at a temperature of $4^{\circ} \mathrm{C}$ throughout the entire experiment, reflecting the normal ambient temperatures at which lobsters are held during fall and winter storage. In warmer waters, we anticipate that a more rapid growth rate of Anophryoides haemophila would enhance parasite susceptibility to the effects of the chemotherapeutants tested, although we did not investigate this in the current study.

Compounds for initial validation of the assay were selected according to a number of criteria. We chose compounds with different mechanisms of anti-protozoal effects and compounds that we had tested in our previous in vitro system (Novotny et al. 1996). Regula- 
tory approval for use in lobsters or in other foodproducing species was also a criterion for selection.

Formaldehyde is used in a bath and is unlikely to have any effect on internal parasites (Speare et al. 1996), but may be useful in reducing external parasite loads. Anophryoides haemophila appeared to be quite resistant to formaldehyde, requiring concentrations greater than $500 \mu \mathrm{M}$ before any significant inhibitory effects were observed. The apparently anomalous increase in parasite numbers after $48 \mathrm{~h}$ incubation with $500 \mu \mathrm{M}$ formaldehyde was not rigorously investigated in this study. Although the ciliates were non-motile, we have observed such ciliates to recover motility and be viable. The increased conversion observed in the tetrazolium assay may have reflected a rebound recovery of A. haemophila after volatilization or degradation of formaldehyde.

In previous experiments in which motility and morphology were used as indicators of efficacy, concentrations of $1 \mathrm{mM}$ formaldehyde were required to produce significant changes in motility and morphology after $4 \mathrm{~h}$ (Novotny et al. 1996), and $1.7 \mathrm{mM}\left(50 \mathrm{mg} \mathrm{l}^{-1}\right.$ ) was required to eliminate motility after a $1 \mathrm{~h}$ exposure (Speare et al. 1996). However, in our experiments, although formaldehyde did have immediate effects, they were much greater at $24 \mathrm{~h}$ and, as indicated by the above results at $48 \mathrm{~h}$ with a concentration of $500 \mu \mathrm{M}$, loss of motility alone is not necessarily an indicator of death. Thus, results obtained using motility assessment at short time periods may not be directly related to actual ciliate death. Nevertheless, the combined results clearly indicate that concentrations of $1 \mathrm{mM}$ or above of formaldehyde would likely be required to be effective in an in vivo setting. Exposure of lobsters for $1 \mathrm{~h}$ to $6.7 \mathrm{mM}$ formaldehyde was not associated with significant adverse behavioural or biochemical effects (Speare et al. 1996), suggesting that these concentrations may be applicable in practice.

Oxytetracycline is one of the few therapeutants approved for use in lobsters in North America. It is licensed for the treatment and prevention of gaffkemia, a bacterial disease. Oxytetracycline is an antibiotic and has limited activity against some protozoal organisms (Riviere \& Spoo 1995). Concentrations of $1 \mathrm{mM}$ can be obtained in lobster hemolymph after exposure to oxytetracycline-containing feed (Bayer \& Daniel 1987). However, in vitro exposure to oxytetracycline for $24 \mathrm{~h}$ produced only minimal inhibition of Anophryoides haemophila growth. Thus, it is unlikely that oxytetracycline will have a significant therapeutic effect in lobsters infected with $A$. haemophila.

Previous in vitro studies of a combination of pyrimethamine and sulphaquinoxaline (Novotny et al. 1996) showed that concentrations of $42.3 \mu \mathrm{g} \mathrm{ml} \mathrm{m}^{-1}$ (40 $\mu \mathrm{M}$ pyrimethamine/110 $\mu \mathrm{M}$ sulphaquinoxaline) were required to alter parasite motility and morphology after a $4 \mathrm{~h}$ exposure. Combinations of sulphonamides and dihydropyrimidine compounds, which act synergistically to inhibit folate synthesis, are known to have good anti-protozoal activity (Spoo \& Riviere 1995). The ratio of the 2 compounds may play an important role in determining efficacy. In our studies, sulphaquinoxaline concentrations above $500 \mu \mathrm{M}$ were required to produce cytotoxicity, while pyrimethamine was effective at concentrations 10 -fold less. Contrary to our expectations, combining the 2 drugs did not improve efficacy. The efficacy of the combination product could be attributed primarily to pyrimethamine once it exceeded concentrations of $50 \mu \mathrm{M}$ (Fig. 7). There would likely be little benefit from using a combination product in the treatment of Anophryoides haemophila infections in lobsters.

Lasalocid is a divalent polyether ionophore antibiotic (Lindsay \& Blagburn 1995), related to monensin, and has activity against several different stages of coccidia. It is approved for use in cattle and chickens, with no required withdrawal period. Lasalocid was highly effective against Anophryoides haemophila in the in vitro assay, producing nearly $100 \%$ ciliate death at concentrations above $50 \mu \mathrm{M}$.

The tetrazolium-based assay described in this report provides a rapid, objective assessment of the effects of compounds on the viabiliy of Anophryoides haemophila as assessed by a metabolic endpoint. The magnitude of the conversion of MTS to a soluble formazan is a combination of the metabolic activity of the ciliates and ciliate number. The results obtained here were consistent with those of a previous assay that was more laborious and subjective. The tetrazolium-based assay is superior in that it is objective, semi-automated, and is able to distinguish between non-motile ciliates and dead ciliates. Results obtained with compounds with different mechanisms of action were consistent with our previous results and, in general, with the anticipated results based on the known activity of the compounds against protozoa. Two compounds, lasalocid and pyrimethamine, may warrant further investigation for in vivo efficacy.

Acknowledgements. This work was supported by an internal grant from the Atlantic Veterinary College and with operating funds provided to the Lobster Health Research Centre by the Max Bell Foundation.

\section{LITERATURE CITED}

Bayer RC, Daniel PC (1987) Safety and efficacy of oxytetracycline for control of gaffkemia in the American labster (Homarus americanus). Fish Res 5:71-81

Cawthorn RJ (1997) Overview of 'bumper car' disease-im- 
pact on the North American lobster fishery. Int J Parasitol $27: 167-172$

Cawthorn RJ, Lynn DH, Despres B, MacMillan R, Maloney R, Loughlin M, Bayer R (1996) Description of Anophryoides haemophila n. sp. (Scuticociliatida: Orchitophryidae), a pathogen of American lobsters Homarus americanus. Dis Aquat Org 24:143-148

Dungan CF, Hamilton RM (1995) Use of a tetrazolium-based cell proliferation assay to measure effects of in vitro conditions on Perkinsus marinus (apicomplexa) proliferation. J Euk Microbiol 42:379-388

Lindsay DS, Blagburn BL (1995) Antiprotozoan drugs. In: Adams HR (ed) Veterinary pharmacology and therapeutics. Iowa State University Press, Ames, p 955-983

Lee JJ, Soldo AT (1992) Protocols in protozoology. Society of Protozoologists, Lawrence, KS, p A-50.5

Marshall NJ, Goodwin CJ, Holt SJ (1995) A critical assessment of the use of microculture tetrazolium assays to measure cell growth and function. Growth Regulation 5:69-84

Messick GA, Small EB (1996) Mesanophrys chesapeakensis

Editorial responsibility: Timothy Flegel,

Bangkok, Thailand n. sp., a histophagous ciliate in the blue crab, Callinectes sapidus, and associated histopathology. Invertebr Biol 115: $1-12$

Novotny MJ, Cawthorn RJ, Despres B (1996) In vitro effects of chemotherapeutants on the lobster parasite Anophryoides haemophila. Dis Aquat Org 24:233-237

Riviere JE, Spoo JW (1995) Tetracycline antibiotics. In: Adams HR (ed) Veterinary pharmacology and therapeutics. Iowa State University Press, Ames, p 784-796

Speare DJ, Cawthorn RJ, Horney BS, MacMillan R, MacKenzie AL (1996) Effects of formalin, chloramine-T, and low salinity dip on the behavior and hemolymph biochemistry of the American lobster. Can Vet J 37:729-734

Spoo JW, Riviere JE (1995) Sulfonamides. In: Adams HR (ed) Veterinary pharmacology and therapeutics. Iowa State University Press, Ames, p 753-773

Vistica DT, Skehan P, Scudiero D, Monks A, Pittman A, Boyd MR (1991) Tetrazolium-based assays for cellular viability: a critical examination of selected parameters affecting formazan production. Cancer Res 51:2515-2520

Submitted: September 14, 1998; Accepted: November 27, 1998 Proofs received from author(s): February 22, 1999 